\title{
ZUR WASSERVERSORGUNG DER MICROCHIROPTEREN EPTESICUS ZULUENSIS VANSONI (VESPERTILIONIDAE) UND SAUROMYS PETROPHILUS ERONGENSIS (MOLOSSIDAE) IN DER NAMIBWƯSTE
}

\author{
von \\ H. ROER, BONN \\ Zoologisches Forschungsinstitut und Museum A. Koenig, 53 Bonn, B.R. Deutschland
}

Während eines vorwiegend entomologischen Untersuchungen in der südlichen Namibwüste gewidmeten Forschungsaufenthaltes von Ende Dezember 1968 bis Mitte März 1969 wurde die Frage untersucht, ob die am mittleren Kuiseb vorkommenden insektenfressenden Fledermäuse auch bei völligem Fehlen von Trinkwasser überleben oder ob Wassermangel sie zur Abwanderung, etwa ins benachbarte Hochland, zwingt.

Die Untersuchungen wurden an der etwa 56 km von der Atlantikküste entfernten Wüstenforschungsstation Gobabeb $(23,3 \mathrm{~S}, 15, \mathrm{O}$ E, $408 \mathrm{~m}$ über NN) mit am gleichen Ort gefangenen Fledermäusen durchgeführt. In Gobabeb treffen 3 Landschaftstypen zusammen: Die nahezu vegetationslose ebene Steinwüste, die mit Ausnahme einzelner Aristida-Büsche ebenfalls pflanzenlose Dünenlandschaft und das Trockenflussbett des Kuiseb mit seinem stellenweise galerieartigen Baumbestand. Der dichte Pflanzenbewuchs im Kuisebflussbett erklärt sich aus der ständigen Grundwasserzufuhr vom benachbarten Hochland.

Obwohl die südliche Namib nahezu niederschlagsfrei ist (nach Schulze, 1969, lag der Regenniederschlag in Gobabeb von Oktober 1962 bis September 1967 bei insgesamt $24 \mathrm{~mm}$, Nebelbildung wurde an 40 Tagen gemessen, die relative Luftfeuchtigkeit variierte im Durchschnitt zwischen $60 \%$ im Februar und $36 \%$ im Mai, die maximale Temperatur betrug $43^{\circ} \mathrm{C}$, die niedrigste $2,1^{\circ} \mathrm{C}$ ), zeichnet sich das mittlere Kuisebtal durch ein für Wüstengebiete bemerkenswert reiches Insektenleben aus. Unsere Lichtfänge an der Wüstenstation haben gezeigt, dass es bei günstigen Witterungsbedingungen nachts zu Massenflügen von Coleopteren, Lepidopteren und Hymenopteren kommen kann. Insektenfressenden Chiropteren stehen somit zu dieser Jahreszeit Beutetiere in ausreichender Menge zur Verfügung. Es stellt sich jedoch die Frage, wie diese Namibfledermäuse ihren Wasserbedarf decken, insbesondere während der Zeit der Jungenaufzucht.

Im Untersuchungsgebiet wurden folgende Microchiropteren nachgewiesen:

1. Eptesicus zuluensis vansoni (Vespertilionidae)

2. Sauromys petrophilus erongensis (Molossidae)

3. Nycteris thebaica damarensis (Nycteridae)

4. Eptesicus hottentotus pallidior (Vespertilionidae)

\section{BEOBACHTUNGEN AN KUNSTLICH ANGELEGTEN WASSERSTELLEN}

An der Vogeltränke der Wüstenstation wie auch an Viehtränken in der weiteren Umgebung stellten wir gelegentlich Fledermäuse fest, die in tiefem Anflug nach Schwalbenart im Fluge Wasser schöpften. Dabei war ein schnappendes Geräusch deutlich wahrnehmbar. In der Regel folgten mehrere Anflüge dicht aufeinander. Es tauchten aber auch Fledermäuse an diesen Wasserstellen auf, die es offensichtlich auf anfliegende Insekten, vor allem Noctuiden, abgesehen hatten. An diesen künstlichen Wasserstellen konnten wir eine Anzahl Fledermäuse mit dem Spiegelnetz einfangen.

\section{UNTERSUCHUNGEN ZUM TRINKWASSERBEDARF}

Um nachzuweisen, wie sich Trinkwasserentzug sowie Aufenthalt bei hohen Temperaturen und gleichzeitig niedriger Luftfeuchtigkeit bei Wüstenfledermäusen auswirkt, wurden $E$. zuluensis vansoni und $S$. petrophilus erongensis einzeln in kleinen Kästchen untergebracht und dem Wüstenklima in Gobabeb direkt ausgesetzt. Dieser Hitzeund Trockenheitsbelastung dürften die Fledermäuse in ihren natürlichen Tagesschlafplätzen 

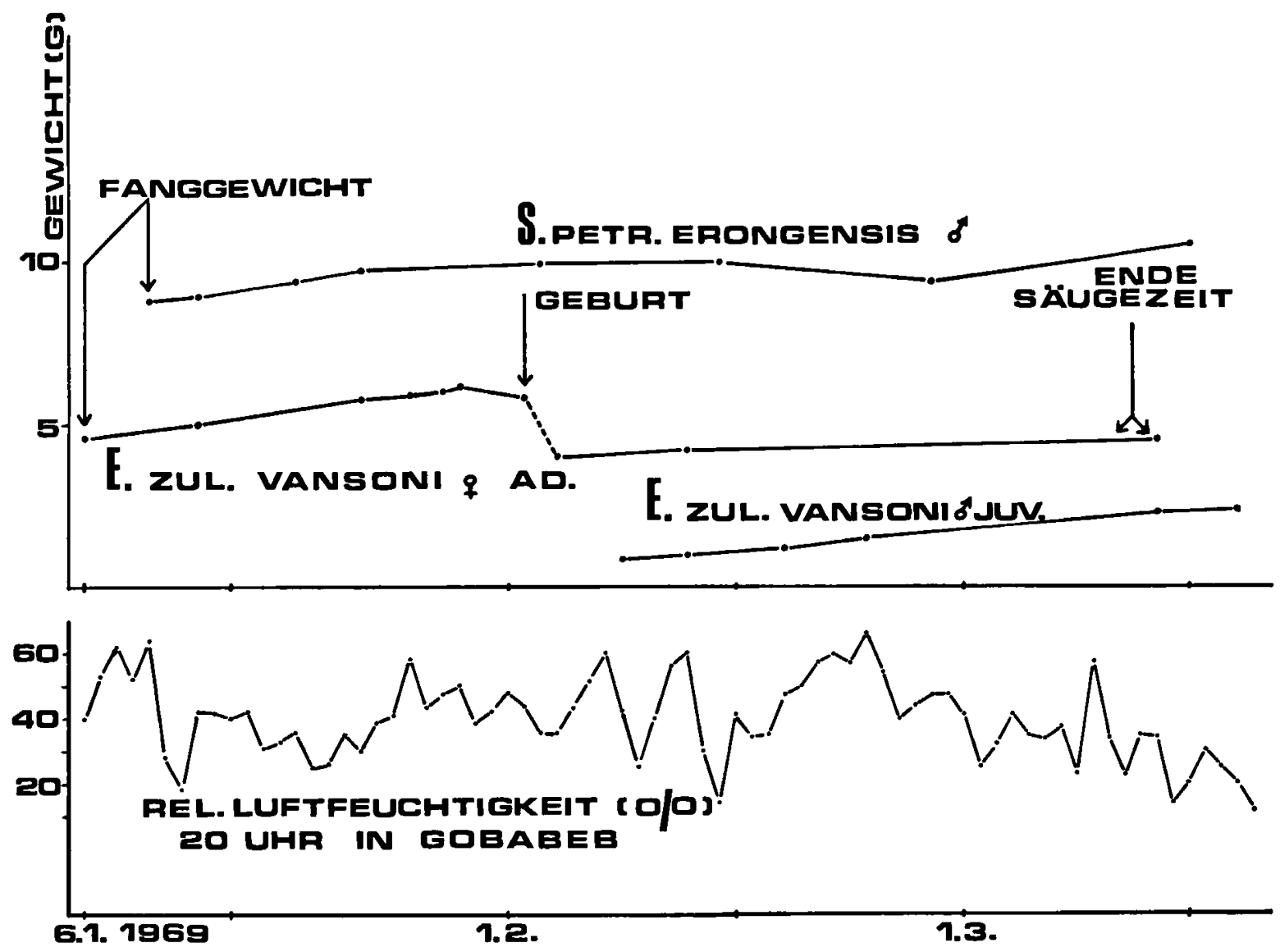

Fig. 1. Körpergewichte verschiedener ohne Trinkwasser in Gobabeb gehaltener Wüstenfledermäuse (oben) und relative Luftfeuchtigkeit um $20 \mathrm{Uhr}$ während der Kontrollzeit (unten).

(Felsspalten, Baumhöhlen) normalerweise nicht ausgesetzt sein. Während der Versuchsperiode vom 6.I.-19.III.1969 hatte Gobabeb an 5 Tagen Morgennebel. Mit $11 \%$ errechte die rel. Luftfeuchtigkeit am 19.III. ihren niedrigsten Wert, während die maximale Temperatur $40^{\circ} \mathrm{C}$ betrug. Die Versuchstiere wurden mit am Licht gefangenen Noctuiden, Cerambyciden, Bostrychiden usw. gefüttert; sie erhielten weder Trinkwasser, noch kamen sie mit Tau oder Nebel in Kontakt. Zur Gewichtsbestimmung, die jeweils nach der Fütterung vorgenommen wurde, diente eine elektrische Waage (Mettler/Zürich).

\section{EXPERIMENT}

Ein am 10.I. gefangenes Männchen von S. petrophilus erongensis wog 8,7 g. Es hat sein Körpergewicht trotz Trinkwasserentzug in den folgenden Wochen nicht nur gehalten, sondern beträcht- lich vergrössert. Am 15.III. hat es vorübergehend ein Körpergewicht von 14,6 g errecht (Fig. 1). Nach Abschluss der Versuche in Gobabeb wurde es in Bonn vom 14.IV-22.X.1969 in einer Klimakammer unseres Instituts bei $29^{\circ} \mathrm{C}$ und $24 \%$ rel. Luftfeuchtigkeit gehalten und dabei ausschliesslich mit Mehlwürmern gefüttert. Die tägliche Nahrungsmenge betrug 6 bis 10 ausgewachsene molitor-Larven. Anfang Oktober machte sich bei dem Tier mangelnde Fresslust und zunehmende Gewichtsabnahme bemerkbar. Am 20.X. betrug sein Körpergewicht noch 9,7 g. Am 22.X. in normale Zimmertemperatur und -feuchtigkeit gebracht, lebte es noch bis zum 31.X. An diesem Tag hatte sich an der Penisspitze eine weisse Salzkruste abgesetzt. Die Bestimmung der Salzkonzentration des Urins hat dankenswerterweise Herr Dr. H. E. Schaefer vom Pathologischen Institut der Universität Köln übernommen. 


\section{EXPERIMENT}

Ein am 6. Januar gefangenes Weibchen von $E$. zuluensis vansoni erwies sich als gravid (Fanggewicht 4,6 g). Es bekam am 2. Februar Zwillinge, von denen eines nach 10 Tagen im Wachstum zurückblieb und am 24. Lebenstag einging. Demgegenüber wurde das zweite Junge von der Mutter bis Ausgang der Laktationsperiode gesäugt (Fig. 1). Mitte März nahm das Muttertier sein Junges nicht mehr an; es ging 3 Tage später ein, vermutlich, weil wir es versäumt hatten, das Junge rechtzeitig auf Insektennahrung umzustellen.

\section{EXPERIMENT}

Ein weiteres, am 9. Februar gefangenes vansoniWeibchen erwies sich ebenfalls als trächtig. Nach anfänglicher kontinuierlicher Gewichtszunahme von 3,9 auf 4,6 g nahm sein Körpergewicht jedoch wieder ab, ohne dass sich Anhaltspunkte für eine stattgefundene Geburt ergeben hätten. Die Frage, ob die Embryonen resorbiert worden sind, muss somit offenbleiben. Dieses Weibchen lebte bis zum 16. März.

\section{BESPRECHUNG DER ERGEBNISSE}

Die vorliegenden Untersuchungen zeigen, dass die Namib-Wüstenfledermäuse Sauromys petrophilus erongensis und Eptesicus zuluensis vansoni während der heissen Jahreszeit mehrere Monate ohne Trinkwasser auskommen können. Bei E. zuluensis vansoni konnte der Nachweis geführt werden, dass unter diesen Lebensbedingungen Jungenaufzucht möglich ist. Demnach können sich Microchiropteren der Namib auch dann behaupten, wenn der Kuiseb infolge Ausbleibens von Niederschlägen im Quellgebiet (Khomashochland) zu keiner Zeit während des Sommers Oberflächenwasser führt und Nebeltage ausbleiben. Die ge- nannten Wüstenfledermäuse müssen demnach das zur Aufrechterhaltung ihrer Lebensfunktionen notwendige Wasser ihren Beutetieren entziehen können, wobei zu untersuchen bleibt, ob sie sowohl das chemisch gebundene als auch freie Wasser verwerten. Bekanntlich schwankt der Anteil an nicht chemisch gebundenem $\mathrm{H}_{2} \mathrm{O}$ bei Insekten in den weiten Grenzen zwischen 45 und 92\% ihres Körpergewichtes. Er ist besonders niedrig bei stark gepanzerten Kerbtieren (z.B. Calandra granaria $46-47 \%$.

\section{SUMMARY}

On the central Kuiseb in the southern Namib insectovorous bats are to be found at an important density. The following species could be approved: Eptesicus hottentotus pallidior (Vespertilionidae), Eptesicus zuluensis vansoni (Vespertilionidae), Sauromys petrophilus erongensis (Molossidae) and Nycteris thebaica damarensis (Nycteridae).

Under the extreme climatic conditions the microchiroptera find prey enough, at least during the summer months, because this dry river-bed with its trees that partially are compact like a forest, offers favourable conditions of development to insects (above all to Coleoptera, Lepidoptera and Hymenoptera). It is being examined in which way these desert bats provide for their need of water in a region where we hardly find open water places during a large period of year. Feeding experiments have shown that Eptesicus zuluensis vansoni and Sauromys petrophilus erongensis can do without having water for several months if they find enough insects. During the observation period a female of $E$. zuluensis vansoni gave birth to twins, one young of which was nourished up to the end of the lactation period.

\section{LITERATUR}

Allen, G. M., 1939. Bats : 1-368. (Dover Publications, New York).

KIRMIZ, J. P., 1962. Adaptation to desert environment : 1-168 (Butterworth \& Co., London).

Schmidt-Nielsen, K. \& B. SChMidT-Nielsen, 1952. Wa- ter metabolism of desert animals. Psysiol. Rev., 32 (2) : 135 .

Schulze, B. R., 1969. The climate of Gobabeb. Sci. Pap. Namib Desert Res. Stn., 37-53 : 5-12.

ShorTRIDGE, G.C., 1934. The mammals of South West Africa, $1: 1-437$. (W. Heinemann Ltd., London). 